\title{
Enumerating preferred extensions: A case study of human reasoning
}

\author{
Alice Toniolo ${ }^{1}$, Timothy J. Norman ${ }^{2}$, and Nir $\mathrm{Oren}^{3}$ \\ 1 School of Computer Science, University of St Andrews, Scotland, UK \\ 2 Department of Electronics and Computer Science, University of Southampton, UK \\ 3 Department of Computing Science, University of Aberdeen, Scotland, UK
}

\begin{abstract}
This paper seeks to better understand the links between human reasoning and preferred extensions as found within formal argumentation, especially in the context of uncertainty. The degree of believability of a conclusion may be associated with the number of preferred extensions in which the conclusion is credulously accepted. We are interested in whether people agree with this evaluation. A set of experiments with human participants is presented to investigate the validity of such an association. Our results show that people tend to agree with the outcome of a version of Thimm's probabilistic semantics in purely qualitative domains as well as in domains in which conclusions express event likelihood. Furthermore, we are able to characterise this behaviour: the heuristics employed by people in understanding preferred extensions are similar to those employed in understanding probabilities.
\end{abstract}

Keywords: Argumentation, Probabilistic Semantics, User Evaluation

\section{Introduction}

One of the strengths of argumentation theory is its qualitative nature. For example, in Dung's theory, arguments are either within, or outside an extension, and no notion of argument strength is required in order to obtain desirable features — such as reinstatement - from the system. More recently, researchers have begun considering more quantitative frameworks, particularly in the context of probabilistic argumentation (e.g., $[8,10,11,18]$ ), through weighted argumentation systems $[2,7]$ and graduality within argumentation [4]. The immediate question then arises as to whether such quantitative representations appropriately capture human reasoning and intuitions, as well as questions regarding the relationship between formal qualitative representations and human quantitative (or semi-quantitative) reasoning. As a concrete example - which we focus on in this paper - one could view multiple extension semantics, such as the preferred semantics, as capturing different possible worlds. This would then suggest that even qualitative argumentation can capture some notion of uncertainty.

This view can be further extended by considering situations where the arguments within an extension are themselves about uncertain facts, effectively changing the likelihood of each extension. If this is the case, then even in 
purely qualitative domains (represented through logical argumentation), where no quantified information exists, the degree of acceptability of a conclusion is associated with the number of preferred extensions in which the conclusion is credulously accepted. This paper investigates the validity of this claim, by means of an experiment with human participants.

The remainder of the paper is structured as follows. In Section 2, we expand the motivations of this work. In Section 3, we introduce an ASPIC-like argumentation framework followed by an overview of its use and key assumptions underpinning our experiments (Section 4). Section 5 details our experimental settings. In Section 6, methodology, hypotheses and results are discussed. We present our conclusions in Section 8.

\section{Background and motivation}

Haenni [8] considers uncertainty as being an evaluation of probability on the premises which propagates throughout the argumentation system. Similarly, other studies such as [18] and [15] model uncertainty on the premises as being associated with the uncertainty of the sources, in the latter case due to the different degrees of trustworthiness of the sources themselves. Li et al [11] consider a different take on probability, namely that the probability of an argument represents a prediction on how likely it is that the argument is justified.

In this work, we are interested in studying the links between the preferred extensions as used in argumentation, and how these are interpreted as probabilities by people with regards to the acceptability of a conclusion. Let us consider a conclusion of an argument within a structured argumentation framework. Generally, argumentation frameworks presented in the literature use extensions to decide whether a conclusion is accepted. In purely qualitative argumentation frameworks, this acceptance is either credulous (when there is at least one extension in which the argument under consideration is accepted), or sceptical (when the argument is accepted in all extensions) [13]. As dictated by the nature of qualitative frameworks, the enumeration of extensions in which a conclusion is accepted does not influence the decision as to whether a conclusion is accepted. However, here we claim that the number of extensions in which a conclusion is accepted has an effect on deciding whether the conclusion is to be considered justified, even if the argumentation framework is fully qualitative ${ }^{1}$.

The problem of understanding the role of enumeration of extensions has been studied by Thimm [16] in abstract argumentation. Thimm presents a novel argumentation framework in which a probabilistic semantics is used to associate an argument with a degree of belief. This belief is computed as function of the number of extensions in which the argument appears to be justified. In our work, we use a similar approach where we consider the enumeration of preferred

\footnotetext{
${ }^{1}$ Note that we use the terms argument and conclusion somewhat interchangeably as in the work we describe, a specific conclusion was the result of a unique argument. In future work, we will consider situations where multiple arguments may lead to the same conclusion, c.f., the so called universal semantics [6].
} 
extensions in evaluating the believability of a conclusion. Thimm claims that this assessment provides a degree of confidence when selecting an option. Here we want to understand whether this is the case, i.e., whether people actually do use a similar heuristic to make a decision on what conclusions are the most believable. In Thimm's work, a probability is associated with each extension, and this influences the degree of belief placed in an argument. In our study we want to understand whether doing so is comparable to human reasoning with probability.

Unlike Thimm's work, we use structured argumentation frameworks, as we are interested in the believability of conclusions rather than arguments. Our core research question is then as follows: do people agree with the evaluation given by probabilistic interpretation of argumentation semantics? To address this question, we define an ASPIC-like structured argumentation framework from which we can formalise the problem.

\section{An ASPIC-like framework with probabilistic semantics}

In order to identify plausible conclusions, we use a simplified ASPIC-like argumentation framework with ordinary premises and defeasible rules without preferences or undercuts $[13,14]$. We derive the degree of belief in a conclusion obtained by applying argumentation semantics to arguments obtained from the framework, and then considering a probabilistic interpretation of the results.

\subsection{Argumentation framework}

Definition 1. An argumentation system $A S$ is a tuple $\langle\mathcal{L},-, \mathcal{R}\rangle$ where $\mathcal{L}$ is a logical language, ${ }^{-}$is a contrariness function, and $\mathcal{R}$ is a set of defeasible rules. The contrariness function ${ }^{-}$is defined from $\mathcal{L}$ to $2^{\mathcal{L}}$, such that given $\varphi \in \bar{\phi}$ with $\varphi, \phi \in \mathcal{L}$, if $\phi \notin \bar{\varphi}, \varphi$ is called the contrary of $\phi$, otherwise if $\phi \in \bar{\varphi}$ they are contradictory (including classical negation $\neg$ ). A defeasible rule is $\varphi_{0}, \ldots, \varphi_{j} \Rightarrow$ $\varphi_{n}$ where $\varphi_{i} \in \mathcal{L}$.

Definition 2. $A$ knowledge-base $K$ in $A S$ is a subset of the language $\mathcal{L}$. An argumentation theory is a pair $A T=\langle K, A S\rangle$.

An argument $A$ is derived from $K$ of theory $A T$. Let $\operatorname{Prem}(A)$ indicate the premises of $A, \operatorname{Conc}(A)$ the conclusion, and $S u b(A)$ the subarguments:

Definition 3. Given a set of arguments Arg, argument $A \in A r g$ is defined as:

$$
\begin{aligned}
& -A=\{\varphi\} \text { with } \varphi \in K \text { where Prem }(A)=\{\varphi\}, \operatorname{Conc}(A)=\varphi, \operatorname{Sub}(A)=\{\varphi\} . \\
& -A=\left\{A_{1}, \ldots, A_{n} \Rightarrow \phi\right\} \text { if there exists a defeasible rule in } A \text { S.t. Conc }\left(A_{1}\right), \\
& \quad \ldots, \operatorname{Conc}\left(A_{n}\right) \Rightarrow \phi \in \mathcal{R} \text { with Prem }(A)=\operatorname{Prem}\left(A_{1}\right) \cup \cdots \cup \operatorname{Prem}\left(A_{n}\right), \\
& \operatorname{Conc}(A)=\phi \text { and } \operatorname{Sub}(A)=\operatorname{Sub}\left(A_{1}\right) \cup \cdots \cup \operatorname{Sub}\left(A_{n}\right) \cup A .
\end{aligned}
$$

Attacks are defined as those arguments that challenge others, while defeats are those attacks that succeed: 
Definition 4. Given two arguments $A_{A}$ and $A_{B}$ :

- $A_{A}$ rebuts $A_{B}$ on $\operatorname{Arg}_{B^{\prime}}$ iff $\operatorname{Conc}\left(A_{A}\right) \in \bar{\varphi}$ for $A_{B^{\prime}} \in \operatorname{Sub}\left(A_{B}\right)$ such that $A_{B^{\prime}}=\left\{A_{B 1}, \ldots, A_{B n^{\prime \prime}} \Rightarrow \varphi\right\}$.

- $A_{A}$ undermines $A_{B}$ on $\varphi$ iff $\operatorname{Conc}\left(A_{A}\right) \in \bar{\varphi}$ such that $\varphi \in \operatorname{Prem}\left(A_{B}\right)$.

Definition 5. Defeat is a binary relationship Def : Arg $\times$ Arg where a defeat is represented as $\left(A_{A}, A_{B}\right) \in$ Def. An argument $A_{A}$ defeats an argument $A_{B}$ iff: i) $A_{A}$ rebuts $A_{B}$ on $A_{B^{\prime}}$; or ii) $A_{A}$ undermines $A_{B}$ on $\varphi$.

Definition 6. An abstract argumentation framework $A F=(A r g, D e f)$ corresponding to an AT contains the set of arguments Arg as defined in Definition 3 and a set of defeats Def as in Definition 5.

Sets of acceptable arguments (i.e., extensions $\xi$ ) in an $A F$ can be computed according to a semantics. Here we use the preferred semantics. The set of credulous preferred extensions is $\hat{\xi}_{P}=\left\{\xi_{1}, \ldots, \xi_{n}\right\}$, where every $\xi_{i}$ is a maximal set of arguments (with respect to set inclusion) that is conflict free and admissible.

Definition 7. Given an abstract argumentation framework $A F=($ Arg, Def $)$, a set of arguments $S \subseteq A r g$ is conflict-free iff there is no $A_{A}, A_{B} \in S$ such that $\left(A_{A}, A_{B}\right) \in$ Def. An argument $A_{A} \in S$ is admissible iff for every $A_{B}$ such that $\left(A_{B}, A_{A}\right) \in$ Def, there is a $A_{C} \in S$ such that $\left(A_{C}, A_{B}\right) \in$ Def.

\subsection{Probabilistic semantics for an argument theory}

Having described a simple ASPIC-like framework, we now describe how Thimm's probabilistic semantics [16] is used to associate probabilities with conclusions.

The set of all possible sets of arguments is referred to as $\mathcal{K}=2^{A r g}$, and the set of preferred extensions $\hat{\xi}_{P}$ is a subset of $\mathcal{K}$. A probability function of the form $P: 2^{\mathcal{K}} \rightarrow[0,1]$ assigns a probability to each set of possible extensions of $A F$. For $\xi \in \mathcal{K}, P(\xi)$ is the probability that $\xi$ is an extension. For now, we make the assumption that extensions are equiprobable. Then the probability of $\xi$ is:

$$
P(\xi)=\left\{\begin{array}{cc}
1 /\left|\hat{\xi}_{P}\right| & \xi \in \hat{\xi}_{P} \\
0 & \xi \notin \hat{\xi}_{P}
\end{array}\right.
$$

For $P(\xi)$ and argument $A \in A r g$ :

$$
\hat{P}(A)=\sum_{A \in \xi \subseteq A r g} P(\xi)
$$

Given the probability function $P, \hat{P}(A)$ represents the degree of belief that an argument $A$ is in an extension according to $P$.

As Thimm suggests we now have an indication of the degree of belief of each argument that gives a characterisation of the uncertainty which is inherent in the AF. We must define several additional concepts in order to describe the acceptability of conclusions within the argumentation framework. 

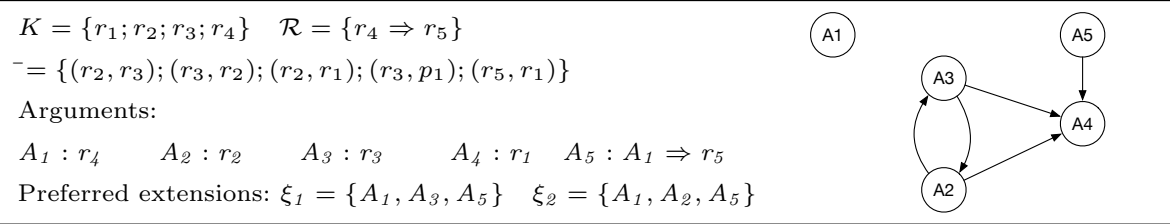

Fig. 1. Example of argumentation theory

From [13] we know that a wff $\varphi \in \mathcal{L}$ is sceptically justified if $\varphi$ is the conclusion of a sceptically justified argument, and credulously justified if $\varphi$ is not sceptically justified and is the conclusion of a credulously justified argument. Hence we define a justification ratio $\mu$ of a conclusion $\varphi$ as follows.

Definition 8. Given a set of arguments $\mathcal{A}=\left\{A_{1}, \ldots, A_{n}\right\}$ such that for any $A_{i}, \operatorname{Conc}\left(A_{i}\right)=\varphi$, we define the justification ratio as $\mu(\varphi)=\sum_{A_{i} \in \mathcal{A}} \hat{P}\left(A_{i}\right)$.

The justification ratio $\mu(\phi)$ captures the probability of a conclusion being justified based on the likelihood of the arguments which justify it. If equiprobable extensions are assumed — as well as unique conclusions for each argument then we obtain:

$$
\mu(\varphi)=\hat{P}(A)=\sum_{A \in \xi \subseteq A r g} 1 /\left|\hat{\xi}_{P}\right| \quad \text { where } \varphi \in \operatorname{Conc}(A)
$$

Example 1. We now illustrate the framework with the following example. Consider the $A T$ presented in Figure 1. We obtain two preferred extensions $\xi_{1}, \xi_{2}$ with $P\left(\xi_{1}\right)=P\left(\xi_{2}\right)=0.5$. The justification ratios are then as follows:

$$
\mu\left(r_{1}\right)=0 \quad \mu\left(r_{2}\right)=\mu\left(r_{3}\right)=0.5 \quad \mu\left(r_{4}\right)=\mu\left(r_{5}\right)=1
$$

\section{Characterising reasoning with extensions}

In the previous section, we explored a method to assign a degree of belief to a conclusion (which we denoted as the justification ratio) in relation to the enumeration of extensions by adapting Thimm's probabilistic semantics. Our main objective is to determine whether people agree with these probabilistic semantics; i.e., whether the justification ratio has a correlation with people's opinion of the believability of a conclusion. We believe that this is the case on the basis of the assumption that people's reasoning with extensions may be understood in relation to reasoning with the rules of classical probability. This assumption leads us to a second objective, namely characterising how people rate the believability of a conclusion.

Our analysis is based on the following observations: 
- Classical probability assigns a likelihood to a piece of information $\varphi$ on the basis of the ratio between the number of favourable and unfavourable cases which support or attack the information. Hence, consider a set of possible worlds $W$ and a subset of the worlds $V \subset W$ in which a proposition $r_{i} \in \mathcal{L}$ holds, the probability of $r_{i}$ is as follows.

$$
p\left(r_{i}\right)=\frac{\# \text { of worlds where } r_{i} \text { holds }}{\text { total \# possible worlds }}=\frac{|V|}{|W|}
$$

- Similarly, we can consider the set of preferred extensions $\hat{\xi}_{P}$ as the set of possible explanations of a world, and the degree of belief of a conclusion $r_{i}$ as given by the justification ratio $\mu\left(r_{i}\right)$. Let us refer to the subset of extensions in which $r_{i}$ is acceptable as $\hat{\xi}_{P}^{r_{i}}$. From Definition 8 we obtain the following.

$$
\mu\left(r_{i}\right)=\sum_{A \in \xi \subseteq A r g} 1 /\left|\hat{\xi}_{P}\right|=\frac{\# \text { extensions in which } r_{i} \text { is acceptable }}{\text { total \# extensions }}=\frac{\left|\hat{\xi}_{P}^{r_{i}}\right|}{\left|\hat{\xi}_{P}\right|}
$$

In the above situation, we assume that the information is purely qualitative. However, the information may refer to the likelihood of an event or a fact [1]. For example, an event $E$ described in $r_{i}$ can be subject of a proposition $r_{j}=$ "there is a $\omega$ chance that event $E$ may occur". Continuing with the similarity between reasoning with extensions and reasoning with probability, we also seek to understand the behaviour in the case in which the user is presented with information that is about the likelihood of events, as well as the uncertainty introduced via the possibility of some information being, or not being, inferred. In this case, the believability of a conclusion may be explained by two heuristics depending on whether people consider these as dependent or independent events. The similarity with an argumentation framework outcome can then be established in the former case through the use of conditional probability, or in the latter by using the multiplication law of probability. For this research, we assume that the second heuristic is adopted, resulting in the following observations:

$-\omega$ indicates the probability of the event $p(E)$. Given $p\left(r_{i}\right)$, the probability of $r_{j}$ using the multiplication law for independent events is: $p\left(r_{j}\right)=\omega * p\left(r_{i}\right)$

- Similarly, in an argumentation framework with probabilistic semantics, given the justification ratio $\mu\left(r_{i}\right)$, the justification ratio of $r_{j}$ is: $\mu\left(r_{j}\right)=\omega * \mu\left(r_{i}\right)$

We are now in a position to describe our experiments, designed to determine (1) whether the probabilistic interpretation of argumentation semantics described above represents human reasoning, and (2) whether the similarities observed between probabilistic and argument based reasoning are valid.

\section{Experiment Design}

Our overall objective is to understand whether people agree with the outcome of Thimm's probabilistic semantics. In our experiments, we asked a participant to 
rate the believability of a proposition under different experimental conditions $\alpha$, as defined below. While considering different experimental conditions, we posed the following question to our subjects: "Given the condition $\alpha$, how likely is that you believe $r_{i}$ "? The subjects were asked to respond on a 5-points Likert scale, a commonly used scale for user studies, recorded as user evaluation $u\left(r_{i}\right)$ of a conclusion $r_{i}$ (with 1: Extremely Unlikely - 5: Extremely Likely). Our hypothesis is that there is a positive correlation between the user rating $u_{\mu}\left(r_{i}\right)$ and the justification ratio $\mu\left(r_{i}\right)$. We also hypothesise that there is a positive correlation between the user evaluation of the likelihood of a piece of information $r_{i}$ $u_{p}\left(r_{i}\right)$ - and its associated probability $p\left(r_{i}\right)$. Finally, we show that there is a similarity between the two ratings $u_{p}\left(r_{i}\right)$ and $u_{\mu}\left(r_{i}\right)$.

Definition 9. An experimental condition $\alpha$ is a tuple $\alpha=\langle$ Domain, Scenario, Proposition, Interpretation, Percentage, Fraction, Ratio $\rangle$.

We now define the components of an experimental condition $\alpha$.

\subsection{Two Types of Information}

As discussed in Section 4, information - represented via propositions - can be classified into two categories, or domain types in the context of the experiment.

Domain 1: Purely qualitative propositions $r_{i} \in \mathcal{L}$ in which the text is about a piece of information.

Domain 2: Propositions $r_{j} \in \mathcal{L}$ in which the text is about a piece of information and its probability of occurring.

In the former, we want to demonstrate that even in purely qualitative scenarios, people agree with the outcome of Thimm's probabilistic semantics: that the believability of a conclusion is related to the number of extensions in which that conclusion is accepted. With the latter domain, we want to demonstrate that in scenarios in which conclusions are about the probability of some information, the outcome of the probabilistic semantics is still an important factor in assessing the believability of a conclusion. The two types of propositions lead to two sets of experiments.

\subsection{Scenarios and Propositions}

In the experiments we use seven base scenarios within a social inference domain - inferences drawn from social media information and corroborated with background knowledge to draw potentially unwanted conclusions [12]. While our work is generalisable to other domains, this seemed to lend itself well to the design of the experiments. The base scenarios are derived from reported incidents in the context of sharing political views [9], and location data or temporal information [12]. These base scenarios are built using a combination of arguments from position to know and cause to effect [17]. 
Each scenario is referred to as $X i$ with $1 \leq i \leq 7$ and designed as a set of propositions, where each proposition $r_{j} \in \mathcal{L}$. In order to collect a relatively large amount of data with less cognitive effort for the user, two propositions per base scenario are chosen and tested by a single subject within our experiments. We combine propositions and base scenario using the same notation, writing $X i_{-} j$, where $j=\{0,1\}$ refers to the proposition being tested. For convenience, we call $X i_{-} j$ a scenario. Given 7 base scenarios and 2 propositions, we obtain a total of 14 scenarios.

\subsection{Interpretations}

For each scenario, two interpretations can be made:

At: An interpretation building on the number of extensions in which the conclusion is acceptable (via an argument theory $A T$, with rules and contraries between propositions), in which a justification ratio $\mu\left(r_{i}\right)$ is associated with each proposition $r_{i}$.

Pt: A possible worlds based probabilistic interpretation, in which each proposition $r_{i}$ is associated with a probability $p\left(r_{i}\right)$ of its information being verified.

We associate the justification ratio of a conclusion $r_{i}$ as the outcome of the probability semantics, with the likelihood that that piece of information is verified (e.g., is shared). Given that both interpretations are based on the same set of propositions, the key design link is such that the justification ratio of $r_{i}$ within $A t$ is the same as the probability of $r_{i}$ in $P t$. Given this equivalence, we refer to this ratio as $\tau=\mu\left(r_{i}\right)=p\left(r_{i}\right)$. With two interpretations per scenario, we obtain 28 experimental conditions $\alpha$. The next factors are further characteristics of conditions $\alpha$.

\subsection{Fractions, Percentages, and Ratios}

In Domain 1 (see Section 5.1), the ratio $\tau$ of a proposition is an irreducible fraction varied between $1 / 6$ and $2 / 3$. That is, we ensured that the conclusion occurred in $\tau$ of the extensions. Besides the main objectives of the experiments, we want to show two further properties: that the scenario has limited influence on the results, and that the ratio - rather than the number of extensions - is the key factor that influences user believability ratings. For demonstrating the latter, we introduce redundant equivalent fractions $\gamma$ (e.g., 1/2,2/4,3/6) corresponding to the ratios $\tau$ using experimental conditions with 2,3,4, or 6 extensions. Each scenario $X i_{-} j$ is associated with a fraction $\gamma$.

In Domain 2, we maintain the same fractions $\gamma$ but also introduce another value, $\omega$, representing the likelihood of the event described within the content of a proposition. For example, a proposition $r_{a}=$ "Joe is a Republican" becomes $r_{b}=$ "There is $70 \%$ chance that Joe is a Republican". We vary $\omega$ between $20 \%$ and $80 \%$ and the overall ratio is given by the product $\tau=\gamma * \omega$. Fractions $\gamma$ and percentages $\omega$ in a scenario are associated using different combinations of both low or high $\omega$ and $\gamma$, or high $\omega$ and low $\gamma$ and vice-versa. 


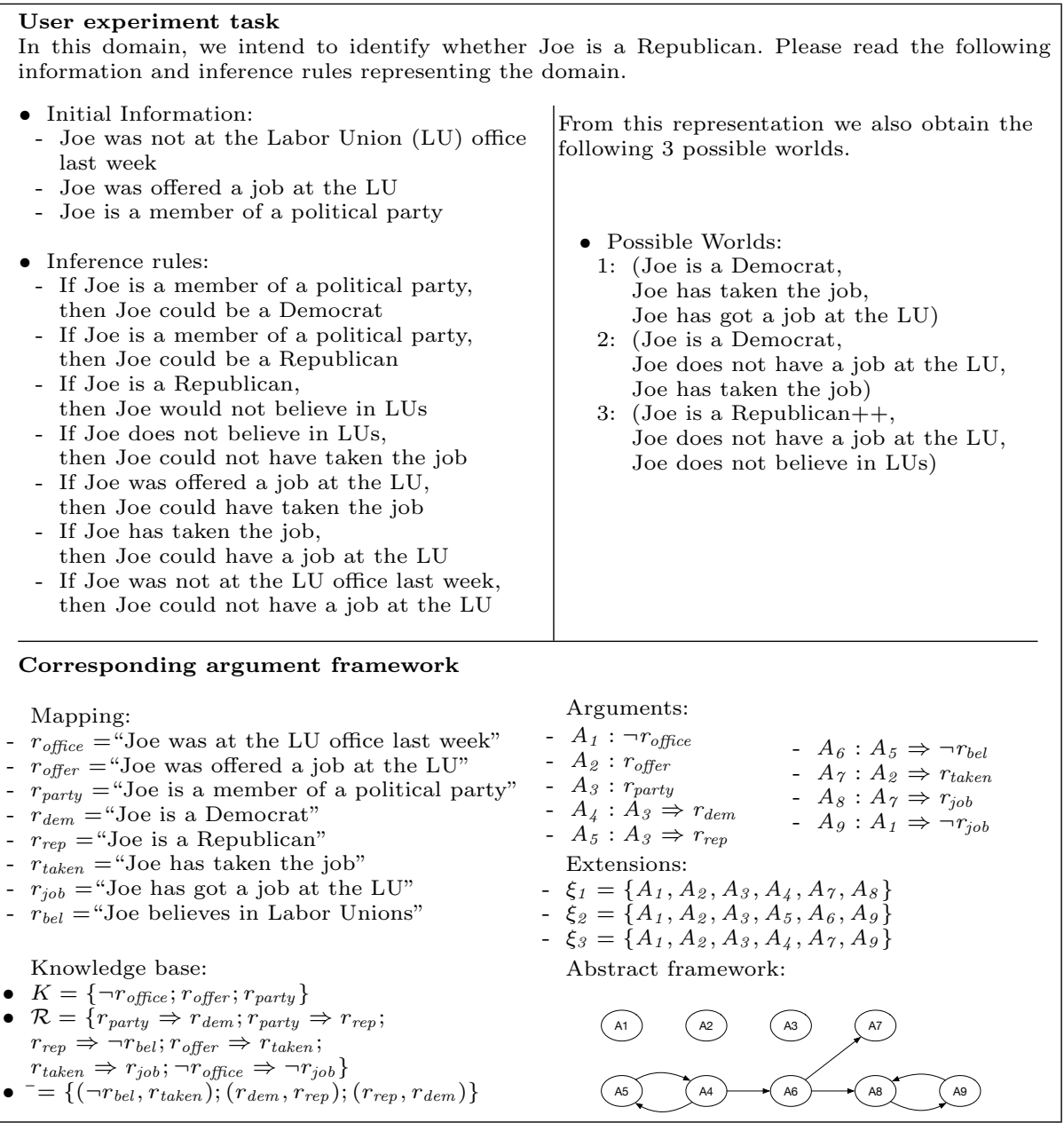

Fig. 2. User Experiment Argument Interpretation \& Framework - Domain 1

Example 2. To obtain an argument theory based interpretation, one of our scenarios presented the user with a set of premises, and grounded defeasible rules from which arguments can be formed. We then only presented the conclusions of arguments from the preferred extensions which result from our framework. For example, Figure 2 presents an example of the argument theory interpretation that is shown to the user during the experiment, and below its correspondent argument framework. In this scenario, 3 preferred extensions existed referred to as possible worlds, and the conclusion $r_{r e p}=$ "Joe is a Republican" is valid in one of these extensions. The experimental condition corresponding to this example is $\alpha_{1}=\left\langle\right.$ Domain:1, Scenario: $X 1 \_1$, Proposition $r_{r e p}$ : "Joe is a Republican", Interpretation:At, Percentage $\omega: 1$, Fraction $\gamma: 1 / 3$, Ratio $\tau: 1 / 3\rangle$. We then asked the user: 


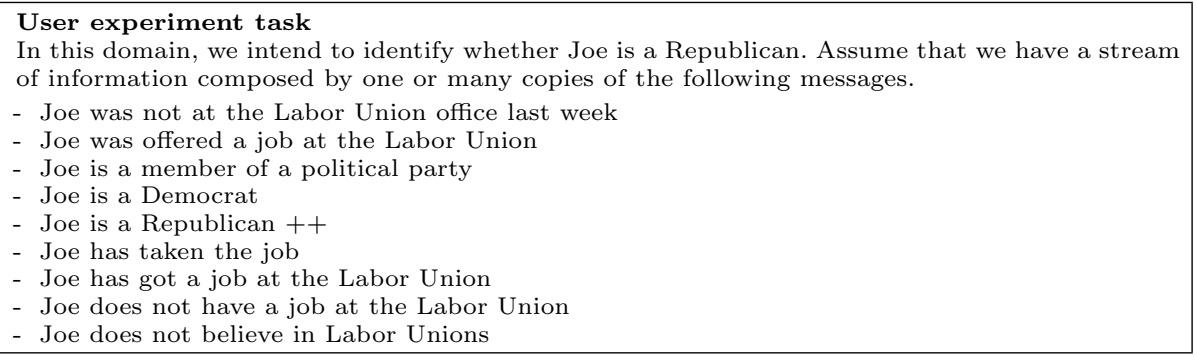

Fig. 3. User Experiment Probabilistic Interpretation - Domain 1

Given the 3 stated possible worlds, how likely is that you would believe that "Joe is a Republican"?

The user's response to the question is recorded as $u_{\mu}\left(r_{r e p}\right)=\{1, \ldots, 5\}$ on a 5-points Likert scale. Assuming that the extensions are equiprobable, we obtain: $P\left(\xi_{1}\right)=P\left(\xi_{2}\right)=P\left(\xi_{3}\right)=1 / 3$ as shown in Figure 2 . The justification ratio for the tested proposition $r_{\text {rep }}$ obtained is $\mu\left(r_{r e p}\right)=1 / 3$.

To obtain a correspondent probabilistic interpretation, we presented the set of propositions to the user as a list of hypothetical messages, which included both premises and conclusions of the above argumentation framework in no particular order. In Figure 3 we present the corresponding experimental scenario. The user was informed that a stream of information would release a number of messages from the list, and asked to comment on the likelihood that a message would state the tested proposition. In this scenario, we also informed the user that 1 out of 3 messages reported that "Joe is a Republican". The experimental condition corresponding to this example is $\alpha_{2}=\left\langle\right.$ Domain:1, Scenario: $X 1 \_1$, Proposition $r_{r e p}$ : "Joe is a Republican", Interpretation:Pt, Percentage $\omega: 1$, Fraction $\gamma: 1 / 3$, Ratio $\tau: 1 / 3\rangle$, where the only difference with $\alpha_{1}$ is the interpretation. To determine $u_{p}\left(r_{r e p}\right)$, the user was asked the question:

If 3 messages are released, how likely is that a message would state that "Joe is a Republican"?.

For these scenarios, $\tau=1 / 3$, and $\gamma=1 / 3$. In the experiments we also tested for situations in which $\tau=1 / 3$, and $\gamma=2 / 6$ for example constructing a similar domain with 6 extensions, where $r_{r e p}$ was valid in only 2 of those. The justification ratio of a proposition in $A t$ corresponds to the probability in $P t$ in Domain 1 such that $p\left(r_{r e p}\right)=\mu\left(r_{r e p}\right)=\gamma=\tau$. In Domain 2, the proposition $r_{\text {prep }}=$ "There is $90 \%$ chance that Joe is a Republican" is used instead, with $\omega=0.9$ in both interpretations and $\omega * p\left(r_{a}\right)=\omega * \mu\left(r_{a}\right)=\omega * \gamma=\tau$. Figure 4 shows an example of the experiment scenario including $r_{\text {prep }}$ for the argument interpretation $A t$. The corresponding probabilistic interpretation $\mathrm{Pt}$ can be derived by extracting all the propositions from this scenario. 


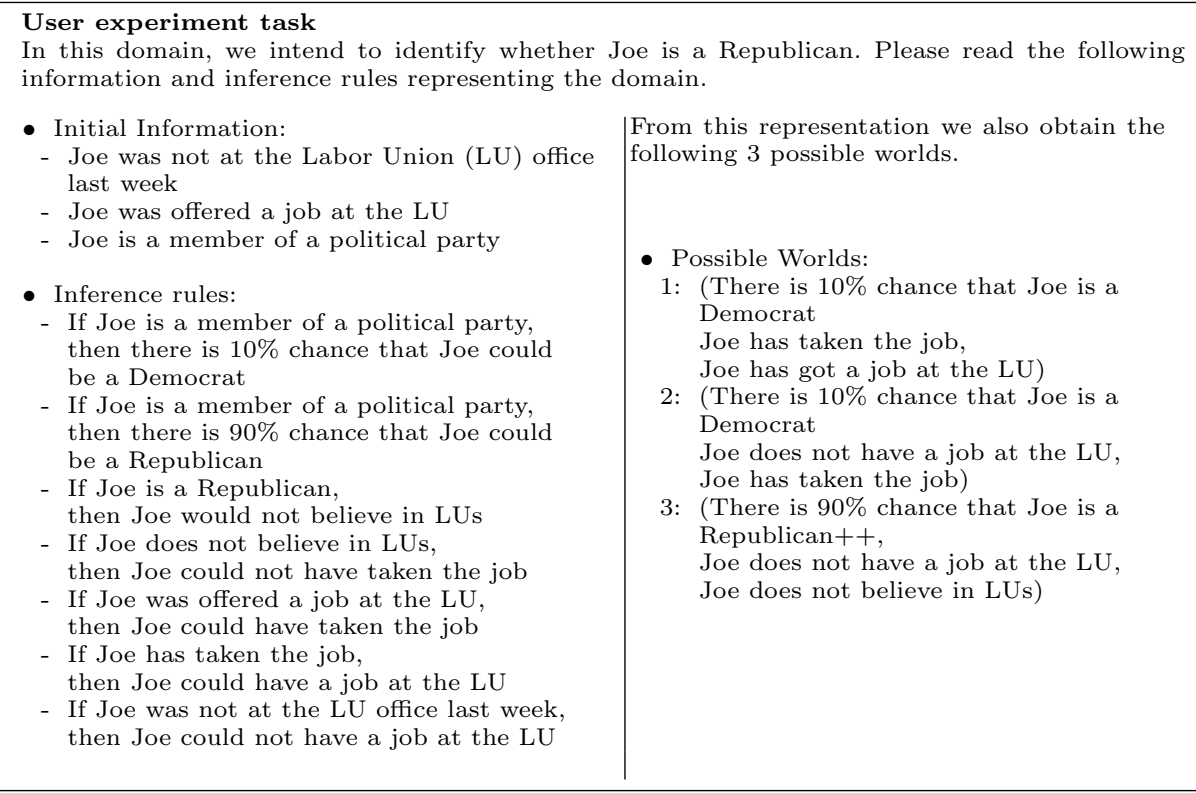

Fig. 4. User Experiment Argument Interpretation - Domain 2

\section{Methodology and results}

We ran our experiments using Amazon Mechanical Turk ${ }^{2}$, a web service that recruits participants to complete tasks. We recruited 420 participants for the experiment from the $\mathrm{USA}^{3}$. Data collection was performed with a questionnaire including four experimental conditions, such that a participant would see two different scenarios, and respond to questions of both problems and interpretations. Initially participants were shown a training example for the argumentation theory to provide them with a basic understanding of argumentation. Each participant was then asked to respond to four combinations of different experimental conditions ( $\alpha$ as described in Section 5).

- Domain 1: two questions within a scenario $X i$, related to conditions $X i_{-} 0$ and $X i_{-} 1$ and an interpretation $A t$ (or $P t$ ).

- Domain 2: two questions within a scenario $X j$, where $i \neq j$, related to conditions $X j_{-} 0$ and $X j_{-} 1$, and an interpretation $P t$ (or $A t$ respectively).

Hence, no user would respond to an interpretation At and its corresponding interpretation $P t$, and each user would see two different domains. We obtained 30 responses per condition $\alpha$. In the remainder of the section, we detail they hypotheses associated with each type of problem, and describe our results.

\footnotetext{
${ }^{2}$ Amazon Mechanical Turk: https://www.mturk.com/

${ }^{3}$ Ethical approval for these experiments was granted by the College Ethics Review Board of the University of Aberdeen on 10/08/2016
} 


\subsection{Domain 1: Hypotheses}

The aim of the first set of experiments is to understand whether people agree with the outcome of the probabilistic semantics when the propositions are purely qualitative. We study the believability rating of a proposition $r_{i}$ in interpretation At as the outcome of the probabilistic semantics $u_{\mu}\left(r_{i}\right)$, and in the corresponding probabilistic interpretation $P t, u_{p}\left(r_{i}\right)$. Our hypotheses are as follows.

H1.1: There is a correlation between the believability rating of $A t, u_{\mu}\left(r_{i}\right)$, and the justification ratio of the conclusions, $\mu\left(r_{i}\right)$, obtained via the outcome of the probabilistic semantics.

H1.2: There is a correlation between the believability rating of $P t, u_{p}\left(r_{i}\right)$, and the probability of the information being verified $p\left(r_{i}\right)$.

H1.3: The two correlations in $A t$ and $P t$ are similar.

We also test the following secondary hypotheses:

H1.4: The scenario does not influence the results: for any two scenarios with the same fraction $\gamma$ there is no difference in the believability rating.

H1.5: The number of extensions does not influence the results: for any two scenarios with same $\tau$ but different $\gamma$ there is no difference in the believability rating.

\subsection{Domain 1: Results}

Figure 5 presents the believability ratings $u_{\mu}\left(r_{i}\right)$ and $u_{p}\left(r_{i}\right)$ recorded for Domain 1. The horizontal axis is ordered according to the fraction $\gamma$ associated with the experimental conditions. We also report the ratio $\tau$ corresponding to the fraction. For each scenario, $u_{\mu}\left(r_{i}\right)$ of $A t$ is shown besides $u_{p}\left(r_{i}\right)$ of $P t$. The graph uses a divergent colour palette; the neutral rating is associated with the brightest colour, ratings below correspond to participants who consider the proposition unlikely, ratings above correspond to those who consider the conclusion likely. Moving from lower to higher $\gamma$ (left to right), we observe that the darker area above the neutral bars increases for both $A t$ and $P t$ interpretations. Within each scenario, the neutral bar is approximately within the same range, with some exceptions. This provides some initial evidence that there is a correlation between the believability ratings and fractions $\gamma$.

A Spearman's rank-order correlation was run for each scenario $X i_{-} j$ to determine the relationship between the believability ratings $u_{\mu}\left(r_{i}\right)$ in $A t$ and the justification ratios $\mu\left(r_{i}\right)=\gamma$, the outcome of the probabilistic semantics. This non-parametric test is used since the results are not normally distributed. The test showed a positive correlation value, $r s$, which was statistically significant $(r s(418)=.288, p \ll 0.001)$. This provides evidence for hypothesis H1.1 — that there is a correlation between the probabilistic semantics and the user believability rating of a conclusion. A similar test determined that there is a statistically significant positive correlation between the believability ratings $u_{p}\left(r_{i}\right)$ in $P t$ and the probabilities $p\left(r_{i}\right)=\gamma(r s(418)=.280, p \ll 0.001)$. This validates hypothesis $\mathrm{H} 1.2$; i.e., there is a correlation between the believability rating of a piece of 


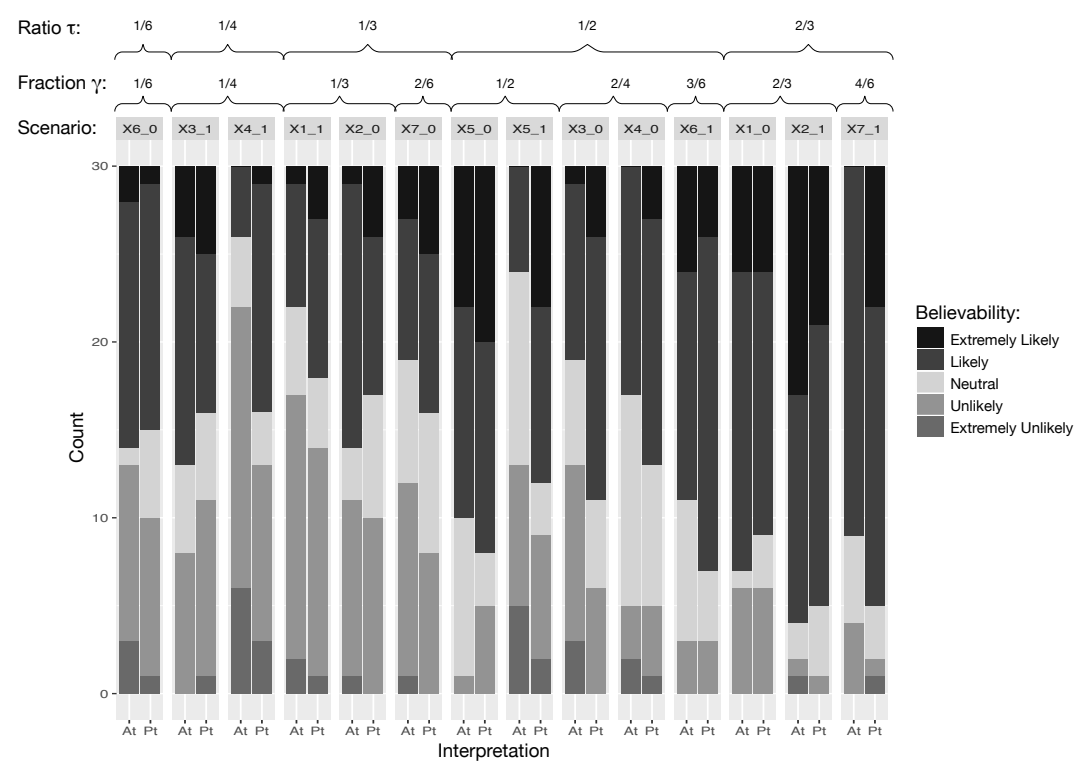

Fig. 5. Believability ratings $u_{\mu}\left(r_{i}\right)$ and $u_{p}\left(r_{i}\right)$ - Domain 1

Table 1. Mann-Whitney U tests on $u_{\mu}\left(r_{i}\right)$ vs. $u_{p}\left(r_{i}\right)$ within scenarios

\begin{tabular}{|l||l|l|l|l|l|l|l|}
\hline Scenario & $X 6 \_0$ & $X 3 \_1$ & $X 4 \_1$ & $X 1 \_1$ & $X 2 \_0$ & $X 7 \_0$ & $X 5 \_0$ \\
\hline p-value & 0.824 & 0.516 & $0.010^{*}$ & 0.265 & 0.888 & 0.247 & 0.744 \\
\hline \hline Scenario & $X 5 \_1$ & $X 3 \_0$ & $X 4 \_0$ & $X 6 \_1$ & $X 1 \_0$ & $X 2 \_1$ & $X 7 \_1$ \\
\hline p-value & $0.005^{*}$ & $0.015^{*}$ & 0.254 & 0.710 & 0.771 & 0.357 & $0.014^{*}$ \\
\hline
\end{tabular}

information and its probability. A comparison between the two correlations was examined using a Fisher's r-to-z transformation. The overall z-score value (based on the difference between the correlations and their variance) was observed to be $z=0.13$ with $p=0.448$. Here, we accept the null hypotheses that the two correlations are not significantly different. This confirms hypothesis H1.3, and characterises how people interpret the outcome of the probabilistic semantics.

There are, however, some outliers that can be noticed in Figure 5. This was investigated with a post-hoc analysis using a series of Mann-Whitney U tests for each scenario $X i_{-} j$ comparing $u_{\mu}\left(r_{i}\right)$ and $u_{p}\left(r_{i}\right)$. Table 1 reports only the p-values, where we consider significance at $p<0.001$. None of the comparisons shows a significant difference, however, for the three scenarios marked with a star, the p-value tends to be low indicating the outliers.

Similar tests are used for the two secondary hypotheses. H1.4 seeks to prove that given the same fraction $\gamma$ (e.g. 1/3), there is no difference between the believability rate of different scenarios associated to that fraction (e.g. $X 1 \_1$ vs. X2_0). In Table 2 we report the p-values of comparisons between different conditions, where significant values are highlighted in bold. Hypothesis H1.4 is 
Table 2. Mann-Whitney U tests on $A t$ and $P t$ between scenarios with similar $\gamma$

\begin{tabular}{|c|c|c|c|c|}
\hline Fraction $\gamma$ & $X_{a}$ & $X_{b}$ & $u_{\mu}\left(r_{i}\right)$ vs. $\mu\left(r_{i}\right)$ & $u_{p}\left(r_{i}\right)$ vs. $p\left(r_{i}\right)$ \\
\hline \hline $1 / 4$ & $X 3 \_1$ & $X 4 \_1$ & 0.403 & $\mathbf{0 . 0 0 0}$ \\
\hline $1 / 3$ & $X 1 \_1$ & $X 2 \_0$ & 0.407 & 0.660 \\
\hline $1 / 2$ & $X 5 \_0$ & $X 5 \_1$ & 0.259 & $\mathbf{0 . 0 0 0}$ \\
\hline $2 / 4$ & $X 3 \_0$ & $X 4 \_0$ & 0.669 & 0.208 \\
\hline $2 / 3$ & $X 1 \_0$ & $X 2 \_1$ & 0.147 & 0.056 \\
\hline
\end{tabular}

Table 3. Mann-Whitney $\mathrm{U}$ tests on $A t$ and $P t$ between scenarios with similar $\tau$

\begin{tabular}{|c|c|c|c|c|}
\hline Ratio $\tau$ & $X_{a}$ & $X_{b}$ & $u_{\mu}\left(r_{i}\right)$ vs. $\mu\left(r_{i}\right)$ & $u_{p}\left(r_{i}\right)$ vs. $p\left(r_{i}\right)$ \\
\hline \hline $1 / 3$ & $X 1 \_1$ & $X 7 \_0$ & 0.201 & 0.187 \\
\hline $1 / 3$ & $X 2 \_0$ & $X 7 \_0$ & 0.629 & 0.579 \\
\hline $1 / 2$ & $X 5 \_0$ & $X 3 \_0$ & 0.147 & $\mathbf{0 . 0 0 1}$ \\
\hline $1 / 2$ & $X 5 \_0$ & $X 4 \_0$ & 0.068 & 0.006 \\
\hline $1 / 2$ & $X 5 \_0$ & $X 6 \_1$ & 0.417 & 0.526 \\
\hline $1 / 2$ & $X 5 \_1$ & $X 3 \_0$ & 0.932 & 0.370 \\
\hline $1 / 2$ & $X 5 \_1$ & $X 4 \_0$ & 0.677 & 0.016 \\
\hline $1 / 2$ & $X 5 \_1$ & $X 6 \_1$ & 0.574 & $\mathbf{0 . 0 0 0}$ \\
\hline $1 / 2$ & $X 3 \_0$ & $X 6 \_1$ & 0.353 & 0.003 \\
\hline $1 / 2$ & $X 4 \_0$ & $X 6 \_1$ & 0.147 & 0.035 \\
\hline $2 / 3$ & $X 1 \_0$ & $X 7 \_1$ & 0.244 & 0.169 \\
\hline $2 / 3$ & $X 2 \_1$ & $X 7 \_1$ & 0.799 & $\mathbf{0 . 0 0 1}$ \\
\hline
\end{tabular}

only partially supported: the scenario tends not to influence the results in $P t$, however, in $A t$, the hypothesis is only supported in 3 out of 5 conditions.

Hypothesis H1.5 focussed on understanding the believability ratings in experimental conditions associated with different fractions $\gamma$ but same ratio $\tau$ (e.g. $1 / 2$ for $X 5 \_0$ vs. $3 / 6$ for $X 6 \_1$ ). In Table 3 we report the p-values for comparisons between these conditions. H1.5 is mainly supported, with the exception of three cases in At. This provides partial evidence that it is the ratio rather than the fraction that influences the believability ratings among different conditions.

\subsection{Domain 2: Hypotheses}

The second problem focusses on understanding whether the outcome of the probabilistic semantics is a factor in assessing the believability of conclusions that are about event likelihood. We hypothesised that the product between the justification ratio of a conclusion and its likelihood influences people's believability ratings in the $A t$ interpretation and is comparable with the multiplication law in the probability interpretation $P t$. We consider similar hypotheses as in Domain 1 , with the difference that the believability rating is now tested for correlation with the product of the fraction $\gamma$ and the likelihood $\omega$ expressed within the content of a proposition $(\tau=\gamma * \omega)$. Hypothesis H2.1 tests for correlation in the interpretation At where $\mu\left(r_{j}\right)=\tau$. Hypothesis H2.2 tests for correlation in $P t$ where $p\left(r_{j}\right)=\tau$ and H2.3 tests for similarity between the two correlations.

\subsection{Domain 2: Results}

Our initial tests study the correlation between the believability ratings and the fractions $\gamma$ or the likelihood $\omega$ alone. Statistical tests were performed using the 


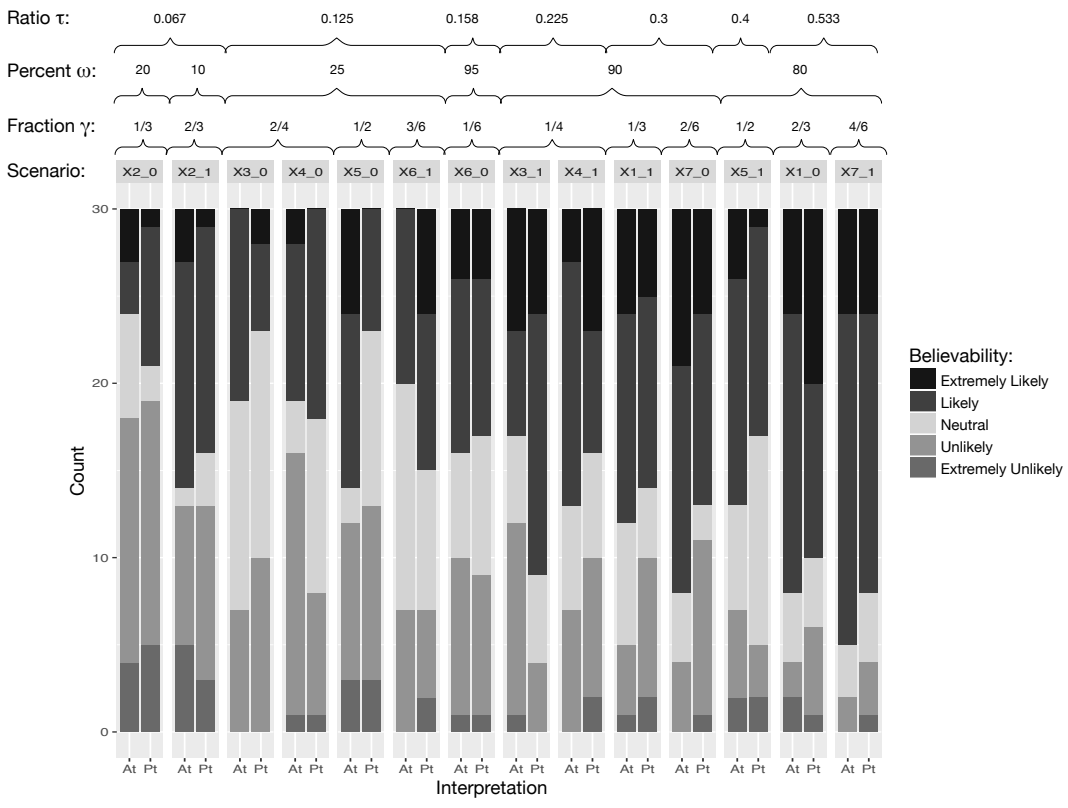

Fig. 6. Believability rating $u_{\mu}\left(r_{i}\right)$ and $u_{p}\left(r_{i}\right)$ - Domain 2

Spearman's rank-order correlation, and similarity is tested using the Fisher's r-to-z transformation, with significance at $p<0.001$. We observed no correlation for fraction $\gamma$ in both interpretations At $(r s(418)=.59, p=0.228)$ and $P t(r s(418)=.26, p=0.596)$. There is, instead, a low correlation with $\omega$ in both At $(r s(418)=.193, p \ll 0.001)$ and $\operatorname{Pt}(r s(418)=.184, p \ll 0.001)$ with high similarity $(z=0.13, p=0.448)$. More interestingly, we found a correlation between the product of $\gamma$ and $\omega$ reflecting the multiplication law of probability in both $A t(r s(418)=.293, p \ll 0.001)$ and $P t(r s(418)=.250, p \ll 0.001)$ with similar behaviour $(z=0.67, p=0.251)$. We now focus on this last result.

In Figure 6, we present the believability rating $u_{\mu}\left(r_{i}\right)$ and $u_{p}\left(r_{i}\right)$ recorded for Domain 2. The horizontal axis is ordered according to $\tau=\gamma * \omega$. The results support hypothesis H2.1 for At: there is a positive correlation between the believability rating and the product of the likelihood expressed within a conclusion and the justification ratio due to the probabilistic semantics. The outcome of the probabilistic semantics is a factor required to interpret the believability ratings: the correlation with the likelihood expressed within a conclusion alone is low $(r s=.193)$ and moderately improves when the product is used $(r s=.293)$. Similar behaviour is observed in Pt supporting H2.2: there is a correlation between the believability rating and the product of the likelihood expressed within the proposition and its probability of occurring. This is stronger than the correlation with the former only ( $r s=.250$ vs. $r s=.184$ ). Finally, H2.3 is supported as no significant difference between the two correlations values is observed. 


\section{Discussion}

We have demonstrated that the outcome of Thimm's probabilistic semantics is an important factor in understanding the believability ratings of the conclusions, even in the case in which a proposition is about the likelihood of an event. The results indicate that people tend to agree with the outcome of the probabilistic semantics. Furthermore, our results confirm that the outcome of the probability semantics may be understood by people in a way similar to the understanding of probability. In the second problem, we showed that this similarity is due to a heuristic associating the product of probabilities to the believability of conclusions. Note that as discussed in Section 4, the multiplication law assumes that there is independence between the event reported by the proposition and it being inferred. We also tested for $\tau$ representing dependent events, using the law of conditional probability. The results showed no correlation with the believability ratings. Due to space constraints, we have omitted these results.

The results of our study are built on a standard (structured) approach to argumentation. While other techniques, such as weighted argumentation could have been used (and will be investigated as future work), we selected the approach used in this paper due to (1) the widely accepted and well understood nature of the standard argumentation semantics; and (2) the ease with which multi-extension semantics from such an approach can be mapped to a many worlds interpretation, from which the comparison to a frequentist probability interpretation can be performed.

The results presented here are - in a sense - preliminary. There are many aspects of this research that need further investigation. To name some, both correlation coefficients are significantly positive but show a moderate correlation between the degree of believability and the justification ratio or associated probability. This suggests that other factors need to be investigated further in the future. One of these aspects is the role of the domains used within the scenarios as we have shown that in the argumentation interpretation this has a more significant role than in the probabilistic view. From an argumentation perspective, further studies should focus on considering other semantics, such as the ranking-based semantics [3]. Further studies should also focus on understanding how people combine probabilities and on analysing human factors, for example, by considering the background of participants involved. We also wish to investigate how cycles and self attacks in the argument graphs, as well as the introduction of preferences may affect our results.

\section{Conclusions}

We investigated whether qualitative argumentation captures some notion of uncertainty by associating a degree of believability of conclusions with the number of preferred extensions. To do so, we examined whether people agree with the outcome of the probabilistic semantics. More broadly, our work can be seen to follow a strand of research similar to that of Cerutti et al. [5], aiming to study the 
alignment between argumentation semantics and human intuition. The novelty of our work is in that we focus on the particular role that multiple extensions play in evaluating the believability of a conclusion.

In this paper, we designed our experiments with a two-fold objective: to determine whether our claim was valid; and to investigate whether there is a similarity between probabilistic and argumentation-based reasoning. Our results show that people tend to agree with the outcome of the probabilistic semantics and that people employ a similar heuristic in understanding both preferred extensions and probabilities. Through our experiments, we obtained some initial promising insights into the use of probability within argumentation frameworks that may guide researchers in better supporting human reasoning in their work.

\section{Acknowledgements}

This work was partially funded by a grant to the University of Aberdeen made by the UK Economic and Social Research Council; Grant reference ES/MOO1628/1.

\section{References}

1. Bailin, S., Battersby, M.: Conductive argumentation, degrees of confidence, and the communication of uncertainty. In: van Eemeren, F.H., Garssen, B. (eds.) Reflections on Theoretical Issues in Argumentation Theory, pp. 71-82. Springer International Publishing (2015)

2. Bench-Capon, T.J.M.: Persuasion in practical argument using value-based argumentation frameworks. Journal of Logic and Computation 13(3), 429-448 (2003)

3. Bonzon, E., Delobelle, J., Konieczny, S., Maudet, N.: A comparative study of ranking-based semantics for abstract argumentation. In: Proceedings of the 30th AAAI Conference on Artificial Intelligence. pp. 914-920 (2016)

4. Cayrol, C., Lagasquie-Schiex, M.C.: Graduality in argumentation. Journal of Artificial Intelligence Research 23(1), 245-297 (2005)

5. Cerutti, F., Tintarev, N., Oren, N.: Formal arguments, preferences, and natural language interfaces to humans: an empirical evaluation. In: Proceedings of the 21st European Conference on Artificial Intelligence. pp. 207-212 (2014)

6. Croitoru, M., Vesic, S.: What can argumentation do for inconsistent ontology query answering? In: Proceedings of the International Conference on Scalable Uncertainty Management. pp. 15-29. Springer (2013)

7. Dunne, P.E., Hunter, A., McBurney, P., Parsons, S., Wooldridge, M.: Weighted argument systems: Basic definitions, algorithms, and complexity results. Artificial Intelligence $175(2), 457-486$ (2011)

8. Haenni, R.: Probabilistic argumentation. Journal of Applied Logic 7(2), 155-176 (2009)

9. Heatherly, R., Kantarcioglu, M., Thuraisingham, B.: Preventing private information inference attacks on social networks. IEEE Transactions on Knowledge and Data Engineering 25(8), 1849-1862 (2013)

10. Hunter, A., Thimm, M.: Probabilistic argument graphs for argumentation lotteries. In: Computational Models of Argument, Frontiers in Artificial Intelligence and Applications, vol. 266, pp. 313-324. IOS Press (2014) 
11. Li, H., Oren, N., Norman, T.J.: Probabilistic argumentation frameworks. In: Modgil, S., Oren, N., Toni, F. (eds.) Theory and Applications of Formal Argumentation, Lecture Notes in Computer Science, vol. 7132. Springer Berlin Heidelberg (2012)

12. Mayer, J.M., Schuler, R.P., Jones, Q.: Towards an understanding of social inference opportunities in social computing. In: Proceedings of the 17th ACM International Conference on Supporting Group Work. pp. 239-248 (2012)

13. Modgil, S., Prakken, H.: The ASPIC+ framework for structured argumentation: A tutorial. Argument \& Computation 5(1), 31-62 (2014)

14. Prakken, H.: An abstract framework for argumentation with structured arguments. Argument \& Computation 1(2), 93-124 (2010)

15. Tang, Y., Cai, K., McBurney, P., Sklar, E., Parsons, S.: Using argumentation to reason about trust and belief. Journal of Logic and Computation 22(5), 979 (2012)

16. Thimm, M.: A probabilistic semantics for abstract argumentation. In: Proceedings of the Twentieth European Conference on Artificial Intelligence. pp. 750-755 (2012)

17. Walton, D., Reed, C., Macagno, F.: Argumentation schemes. Cambridge University Press (2008)

18. Zenker, F.: Bayesian argumentation: The practical side of probability. In: Bayesian Argumentation, Synthese Library, vol. 362, pp. 1-11. Springer (2013) 